\title{
Supersymmetry, quantum corrections, and the higher derivative regularization
}

\author{
Konstantin Stepanyantz ${ }^{1, *}$ \\ ${ }^{1}$ Moscow State University, Faculty of Physics, Department of Theoretical Physics, 119991, Moscow, \\ Russia
}

\begin{abstract}
We investigate the structure of quantum corrections in $\mathcal{N}=1$ supersymmetric theories using the higher covariant derivative method for regularization. In particular, we discuss the non-renormalization theorem for the triple gauge-ghost vertices and its connection with the exact NSVZ $\beta$-function. Namely, using the finiteness of the triple gauge-ghost vertices we rewrite the NSVZ equation in a form of a relation between the $\beta$-function and the anomalous dimensions of the quantum gauge superfield, of the Faddeev-Popov ghosts, and of the matter superfields. We argue that it is this form that follows from the perturbative calculations, and give a simple prescription how to construct the NSVZ scheme in the non-Abelian case. These statements are confirmed by an explicit calculation of the three-loop contributions to the $\beta$-function containing Yukawa couplings. Moreover, we calculate the two-loop anomalous dimension of the ghost superfields and demonstrate that for doing this calculation it is very important that the quantum gauge superfield is renormalized non-linearly.
\end{abstract}

\section{Introduction}

Investigating of $\mathcal{N}=1$ supersymmetric gauge theories is very interesting for both phenomenology and theory. Really, these theories are the main building blocks for constructing supersymmetric extensions of the Standard Model. Due to $\mathcal{N}=1$ supersymmetry it is possible to solve some problems of the Standard Model. Namely, running of the gauge coupling constants in the supersymmetric case agrees with the predictions of Grand Unified Theories. The proton life time is essentially increased. There are no quadratically divergent quantum corrections to the Higgs mass.

From the theoretical point of view the famous feature of supersymmetric theories is cancellation of some ultraviolet divergences. In particular, for $\mathcal{N}=1 D=4$ supersymmetric theories the following non-renormalization theorems exist:

1. There are no divergent quantum corrections to the superpotential [1].

2. The $\beta$-function of $\mathcal{N}=1$ gauge theories is related to the anomalous dimension of the matter superfields by the so-called Novikov, Shifman, Vainshtein, and Zakharov (NSVZ) $\beta$-function [2-5], ${ }^{1}$

*e-mail: stepan@m9com.ru

${ }^{1}$ For a recent discussion see Ref. [6]. 


$$
\beta(\alpha, \lambda)=-\frac{\alpha^{2}\left(3 C_{2}-T(R)+C(R)_{i}{ }^{j} \gamma_{j}{ }^{i}(\alpha, \lambda) / r\right)}{2 \pi\left(1-C_{2} \alpha / 2 \pi\right)},
$$

where $r$ is the dimension of the gauge group, and the other constants are defined by the equations

$$
\operatorname{tr}\left(T^{A} T^{B}\right) \equiv T(R) \delta^{A B} ; \quad\left(T^{A}\right)_{i}{ }^{k}\left(T^{A}\right)_{k}{ }^{j} \equiv C(R)_{i}{ }^{j} ; \quad f^{A C D} f^{B C D} \equiv C_{2} \delta^{A B} .
$$

3. The three-point vertices with two lines of the Faddeev-Popov ghosts and one line of the quantum gauge superfield are finite in all orders [7].

The NSVZ $\beta$-function (obtained from general arguments) was compared with the results of calculations in the lowest orders (up to the four-loop approximation) made with the dimensional reduction [8] in the minimal subtraction scheme (which in this case is called the $\overline{\mathrm{DR}}$-scheme) in Refs. [9-12]. The result coincided with the NSVZ $\beta$-function only in the oneand two-loop approximations. In the higher loops it is necessary to make a special tuning of the coupling constant $[10,11,13]$, which is equivalent to changing the subtraction scheme. Note that the NSVZ relation is scheme-dependent. The general equations which describe its scheme-dependence have been derived in Refs. $[14,15]$. The possibility to restore the NSVZ relation by changing the subtraction scheme is non-trivial [10] and confirms the correctness of the NSVZ equation. However, it raises a problem, how to construct the renormalization prescription which produces the NSVZ result in all orders. At present, in the case of using the dimensional reduction such a prescription has not yet been found.

However, if the theory is regularized by the Slavnov higher covariant derivative method [16-18] in a supersymmetric version $[19,20]$, the problem can be solved in a natural and beautiful way. In the Abelian case the NSVZ relation is obtained in all orders with the higher derivative regularization for the renormalization group functions (RGFs) defined in terms of the bare coupling constant independently of the renormalization prescription [21, 22]. For scheme-dependent RGFs (standardly) defined in terms of the renormalized coupling constant the NSVZ subtraction scheme is obtained with the higher derivative regularization supplemented by the minimal subtraction of logarithms [15, 23, 24]. Such a renormalization prescription we will call HD+MSL [25-27]. ${ }^{2}$ In this paper we will explain, how these results can be generalized to the non-Abelian case and describe some peculiarities of calculating quantum corrections in $\mathcal{N}=1$ supersymmetric gauge theories.

\section{Non-Abelian $\mathcal{N}=1$ supersymmetric gauge theories: action, regularization, and quantization}

In the $\mathcal{N}=1$ superspace the massless non-Abelian $\mathcal{N}=1$ supersymmetric Yang-Mills (SYM) theory with matter is described by the action

$$
S=\frac{1}{2 e_{0}^{2}} \operatorname{Re} \operatorname{tr} \int d^{4} x d^{2} \theta W^{a} W_{a}+\frac{1}{4} \int d^{4} x d^{4} \theta \phi^{* i}\left(e^{2 \mathcal{F}(V)}\right)_{i}^{j} \phi_{j}+\left\{\frac{1}{6} \lambda_{0}^{i j k} \int d^{4} x d^{2} \theta \phi_{i} \phi_{j} \phi_{k}+\text { c.c. }\right\},
$$

where the chiral matter superfields $\phi_{i}$ belong to a representation $R$ of the gauge group, and the Yukawa couplings $\lambda_{0}$ satisfy the condition $\lambda_{0}^{i j m}\left(T^{A}\right)_{m}{ }^{k}+\lambda_{0}^{i m k}\left(T^{A}\right)_{m}{ }^{j}+\lambda_{0}^{m j k}\left(T^{A}\right)_{m}{ }^{i}=0$.

\footnotetext{
${ }^{2}$ The HD+MSL prescription was proved to produce the NSVZ-like schemes for the Adler $D$-function in $\mathcal{N}=1$ SQCD [28, 29] and for the anomalous dimension of the photino mass in softly broken $\mathcal{N}=1$ SQED [30, 31].
} 
Usually, writing this action one sets $\mathcal{F}(V)=V$, but below we will see that for calculating quantum corrections the function $\mathcal{F}(V)$ is very important. In this case the supersymmetric gauge superfield strength is defined by the equation $W_{a} \equiv \bar{D}^{2}\left(e^{-2 \mathcal{F}(V)} D_{a} e^{2 \mathcal{F}(V)}\right) / 8$.

The explicitly gauge invariant effective action is constructed with the help of the background field method, which is introduced by the substitution $e^{2 \mathcal{F}(V)} \rightarrow e^{\mathbf{\Omega}^{+}} e^{2 \mathcal{F}(V)} e^{\mathbf{\Omega}}$. Then the background superfield $\boldsymbol{V}$ is given by the expression $e^{2 \boldsymbol{V}}=e^{\mathbf{\Omega}^{+}} e^{\mathbf{\Omega}}$.

We will regularize the considered theory by the Slavnov higher covariant derivative method. For this purpose we add to the action the higher derivative term

$$
\begin{aligned}
& S_{\Lambda}=\frac{1}{2 e_{0}^{2}} \operatorname{Re} \operatorname{tr} \int d^{4} x d^{2} \theta e^{\mathbf{\Omega}} W^{a} e^{-\boldsymbol{\Omega}}\left[R\left(-\frac{\bar{\nabla}^{2} \nabla^{2}}{16 \Lambda^{2}}\right)-1\right]_{A d j} e^{\mathbf{\Omega}} W_{a} e^{-\boldsymbol{\Omega}} \\
& +\frac{1}{4} \int d^{4} x d^{4} \theta \phi^{+} e^{\mathbf{\Omega}^{+}} e^{2 \mathcal{F}(V)}\left[F\left(-\frac{\bar{\nabla}^{2} \nabla^{2}}{16 \Lambda^{2}}\right)-1\right] e^{\mathbf{\Omega}} \phi .
\end{aligned}
$$

where the regulators $R$ and $F$ have a rapid growth at infinity. The gauge fixing term also contains the higher derivative regulator $K$,

$$
S_{\mathrm{gf}}=-\frac{1}{16 \xi_{0} e_{0}^{2}} \operatorname{tr} \int d^{4} x d^{4} \theta \boldsymbol{\nabla}^{2} V K\left(-\frac{\overline{\boldsymbol{\nabla}}^{2} \boldsymbol{\nabla}^{2}}{16 \Lambda^{2}}\right)_{A d j} \overline{\boldsymbol{\nabla}}^{2} V .
$$

In this case the action for the Faddeev-Popov ghosts has the form

$$
\begin{aligned}
& S_{\mathrm{FP}}=\left.\frac{1}{2} \int d^{4} x d^{4} \theta \frac{\partial \mathcal{F}^{-1}(\widetilde{V})^{A}}{\partial \widetilde{V}^{B}}\right|_{\widetilde{V}=\mathcal{F}(V)}\left(e^{\mathbf{\Omega}} \bar{c} e^{-\mathbf{\Omega}}+e^{-\mathbf{\Omega}^{+}} \bar{c}^{+} e^{\mathbf{\Omega}^{+}}\right)^{A} \\
& \times\left\{\left(\frac{\mathcal{F}(V)}{1-e^{2 \mathcal{F}(V)}}\right)_{A d j}\left(e^{-\mathbf{\Omega}^{+}} c^{+} e^{\mathbf{\Omega}^{+}}\right)+\left(\frac{\mathcal{F}(V)}{1-e^{-2 \mathcal{F}(V)}}\right)_{A d j}\left(e^{\mathbf{\Omega}} c e^{-\mathbf{\Omega}}\right)\right\}^{B} .
\end{aligned}
$$

Also it is necessary to introduce the Nielsen-Kallosh ghosts.

Note that the quantum gauge superfield $V$ is renormalized nonlinearly [32-34]. Parameters describing this nonlinear renormalization are included into the function $\mathcal{F}(V)$. In our notation the renormalization constants are defined by the equations

$$
\begin{aligned}
& V=Z_{V} Z_{\alpha}^{-1 / 2} V_{R}+\text { nonlinear terms; } \quad \lambda^{i j k}=\lambda_{0}^{m n p}\left(Z_{\lambda}\right)_{m}{ }^{i}\left(Z_{\lambda}\right)_{n}{ }^{j}\left(Z_{\lambda}\right)_{p}{ }^{k} ; \\
& \frac{1}{\alpha_{0}}=\frac{Z_{\alpha}}{\alpha} ; \quad \frac{1}{\xi_{0}}=\frac{Z_{\xi}}{\xi} ; \quad \bar{c} c=Z_{c} Z_{\alpha}^{-1} \bar{c}_{R} c_{R} ; \quad \phi_{i}=\left(\sqrt{Z_{\phi}}\right)_{i}{ }^{j}\left(\phi_{R}\right)_{j},
\end{aligned}
$$

where it is possible to choose $\left(Z_{\lambda}\right)_{i}{ }^{j}=\left(\sqrt{Z_{\phi}}\right)_{i}{ }^{j} ; Z_{\xi}=Z_{V}^{-2}$.

\section{Finiteness of triple gauge-ghost vertices, new form of the NSVZ relation, and the NSVZ scheme in the non-Abelian case}

According to Ref. [7] the three-point vertices with two ghost legs and a single leg of the quantum gauge superfield are finite in all orders. In the general $\xi$-gauge this statement has first been noted in the one-loop calculation of Ref. [35]. ${ }^{3}$ In the superfield formulation of

\footnotetext{
${ }^{3}$ Earlier, a similar statement was known only in the Landau gauge $\xi \rightarrow 0$ for the usual (non-sypersymmetric) Yang-Mills theory [36] and for the $\mathcal{N}=1$ SYM in the Wess-Zumino gauge (also in the case $\xi \rightarrow 0$ ) [37].
} 
$\mathcal{N}=1$ supersymmetric gauge theories there are 4 such vertices, $\bar{c} V c, \bar{c}^{+} V c, \bar{c} V c^{+}$, and $\bar{c}^{+} V c^{+}$ which have the same renormalization constant $Z_{\alpha}^{-1 / 2} Z_{c} Z_{V}$. Therefore, the above statement can be rewritten as

$$
\frac{d}{d \ln \Lambda}\left(Z_{\alpha}^{-1 / 2} Z_{c} Z_{V}\right)=0
$$

where $Z_{V}$ describes the renormalization of the quantum gauge superfield in the linear approximation, see Eq. (7). Note that the Green functions of the structure $\bar{c} V^{n} c$ are divergent for $n \neq 1$. The explicit expressions for the Green functions with $n=0$ and $n=1$ in the one-loop approximation can be found in Refs. [35] and [7], respectively. The former Green function is divergent, while the letter ones are finite.

Using the non-renormalization theorem for the triple gauge-ghost vertices the NSVZ equation can be presented in a different form. Writing this equation we will define RGFs in terms of the bare couplings by the equations

$$
\begin{aligned}
& \beta\left(\alpha_{0}, \lambda_{0}\right) \equiv \frac{d \alpha_{0}}{d \ln \Lambda} ; \quad\left(\gamma_{\phi}\right)_{i}{ }^{j}\left(\alpha_{0}, \lambda_{0}\right) \equiv-\frac{d \ln \left(Z_{\phi}\right)_{i}{ }^{j}(\alpha, \lambda, \Lambda / \mu)}{d \ln \Lambda} ; \\
& \gamma_{V}\left(\alpha_{0}, \lambda_{0}\right) \equiv-\frac{d \ln Z_{V}(\alpha, \lambda, \Lambda / \mu)}{d \ln \Lambda} ; \quad \gamma_{c}\left(\alpha_{0}, \lambda_{0}\right) \equiv-\frac{d \ln Z_{c}(\alpha, \lambda, \Lambda / \mu)}{d \ln \Lambda},
\end{aligned}
$$

where the differentiation is made at fixed values of renormalized parameters. These RGFs are scheme independent at a fixed regularization [23], but depend on a regularization. For $\mathcal{N}=1$ SQED with $N_{f}$ flavors regularized by higher derivatives they satisfy the NSVZ relation in all orders $[21,22]$. For these RGFs the NSVZ $\beta$-function can equivalently be rewritten in the form

$$
\frac{\beta\left(\alpha_{0}, \lambda_{0}\right)}{\alpha_{0}^{2}}=-\frac{\left.3 C_{2}-T(R)+C(R)_{i}{ }^{j} \gamma_{\phi}\right)_{j}{ }^{i}\left(\alpha_{0}, \lambda_{0}\right) / r}{2 \pi}+\frac{C_{2}}{2 \pi} \cdot \frac{\beta\left(\alpha_{0}, \lambda_{0}\right)}{\alpha_{0}} .
$$

Using the finiteness of the triple gauge-ghost vertices the $\beta$-function in the right hand side can be presented as

$$
\beta\left(\alpha_{0}, \lambda_{0}\right)=\left.\frac{d \alpha_{0}(\alpha, \lambda, \Lambda / \mu)}{d \ln \Lambda}\right|_{\alpha, \lambda=\mathrm{const}}=-\left.\alpha_{0} \frac{d \ln Z_{\alpha}}{d \ln \Lambda}\right|_{\alpha, \lambda=\mathrm{const}}=2 \alpha_{0}\left(\gamma_{c}\left(\alpha_{0}, \lambda_{0}\right)+\gamma_{V}\left(\alpha_{0}, \lambda_{0}\right)\right),
$$

where $\gamma_{c}$ and $\gamma_{V}$ are the anomalous dimensions of the Faddeev-Popov ghosts and of the quantum gauge superfield, respectively. Substituting this expression into the right hand side of the NSVZ relation we obtain

$$
\frac{\beta\left(\alpha_{0}, \lambda_{0}\right)}{\alpha_{0}^{2}}=-\frac{1}{2 \pi}\left(3 C_{2}-T(R)-2 C_{2} \gamma_{c}\left(\alpha_{0}, \lambda_{0}\right)-2 C_{2} \gamma_{V}\left(\alpha_{0}, \lambda_{0}\right)+C(R)_{i}{ }^{j}\left(\gamma_{\phi}\right)_{j}{ }^{i}\left(\alpha_{0}, \lambda_{0}\right) / r\right)
$$

From this form of the NSVZ $\beta$-function we see that the matter superfields and ghosts similarly contribute to the right hand side.

The graphical interpretation of Eq. (12) is similar to the Abelian case considered in $[38,39]$. Namely, if we start with a supergraph without external lines, then the contribution to the left hand side comes from diagrams obtained by attaching two external legs of the background gauge superfield. The corresponding contributions to the anomalous dimensions of the quantum superfields are obtained by cutting internal lines in the original graph. The new 
form of the NSVZ relation establishes a correspondence between these $n$-loop contribution to the $\beta$-function and $(n-1)$-loop contributions to the anomalous dimensions. The equality takes place due to factorization of loop integrals giving the $\beta$-function into integrals of total $[40,41]$ and even double total derivatives [38, 42].

Because the NSVZ relation in the form (12) has the same graphical interpretation as in the Abelian case, it is reasonable to suggest that as in the Abelian case it is obtained with the higher covariant derivative regularization for RGFs defined in terms of the bare couplings independently of the renormalization prescription. This proposal has been confirmed by explicit calculations of the terms containing the Yukawa couplings in the three-loop $\beta$-function and in the two-loop anomalous dimensions of the quantum superfields $[43,44]$. For the other terms it has been verified in the previous order [45]. However, RGFs defined in terms of the renormalized couplings by the equations

$$
\begin{array}{lr}
\left.\widetilde{\beta}(\alpha, \lambda) \equiv \frac{d \alpha}{d \ln \mu} ; \quad \widetilde{\gamma}_{\phi}\right)_{i}{ }^{j}(\alpha, \lambda) \equiv \frac{d \ln \left(Z_{\phi}\right)_{i}{ }^{j}\left(\alpha_{0}, \lambda_{0}, \Lambda / \mu\right)}{d \ln \mu} ; \\
\widetilde{\gamma}_{V}(\alpha, \lambda) \equiv \frac{d \ln Z_{V}\left(\alpha_{0}, \lambda_{0}, \Lambda / \mu\right)}{d \ln \mu} ; & \widetilde{\gamma}_{c}(\alpha, \lambda) \equiv \frac{d \ln Z_{c}\left(\alpha_{0}, \lambda_{0}, \Lambda / \mu\right)}{d \ln \mu},
\end{array}
$$

where the differentiation is made at fixed values of bare parameters, are scheme and regularization dependent and satisfy the NSVZ relation only for a special renormalization prescription. To construct this (NSVZ) scheme, we note that, similarly to the $\mathcal{N}=1 \mathrm{SQED}$ case considered in [23, 24], for the non-Abelian theories both definitions of RGFs give the same functions, if the boundary conditions

$$
Z_{\alpha}\left(\alpha, \lambda, x_{0}\right)=1 ; \quad\left(Z_{\phi}\right)_{i}{ }^{j}\left(\alpha, \lambda, x_{0}\right)=\delta_{i}{ }^{j} ; \quad Z_{c}\left(\alpha, \lambda, x_{0}\right)=1
$$

are imposed on the renormalization constants, where $x_{0}$ is a certain fixed value of $\ln \Lambda / \mu$. (We also assume that $Z_{V}=Z_{\alpha}^{1 / 2} Z_{c}^{-1}$.) For $x_{0}=0$ this implies that only powers of $\ln \Lambda / \mu$ are included into the renormalization constants, so that the corresponding renormalization prescription is very similar to the minimal subtraction scheme. That is why we will call it HD+MSL, i.e. Higher Derivatives + Minimal Subtractions of Logarithms. If RGFs defined in terms of the bare couplings satisfy Eq. (12) with the higher covariant derivative regularization, then HD+MSL=NSVZ.

\section{Three-loop verification for terms containing the Yukawa couplings}

The above results have been verified in the three-loop approximation for terms containing the Yukawa couplings [43, 44]. For the higher derivative regulators $F(x)=1+x^{n} ; R(x)=$ $1+x^{m}$ RGFs defined in terms of the renormalized couplings are

$$
\begin{aligned}
& \frac{\widetilde{\beta}(\alpha, \lambda)}{\alpha^{2}}=-\frac{1}{2 \pi}\left(3 C_{2}-T(R)\right)-\frac{1}{2 \pi r} C(R)_{j}{ }^{i}\left(\frac{1}{4 \pi^{2}} \lambda_{i m n}^{*} \lambda^{j m n}+\frac{\alpha}{8 \pi^{3}} \lambda_{i m n}^{*} \lambda^{j m n} C_{2}\right. \\
& +\frac{\alpha}{4 \pi^{3}} \lambda_{l m n}^{*} \lambda^{j m n} C(R)_{i}{ }^{l}\left[b_{2}-g_{11}-\frac{1}{2}\left(1-\frac{1}{n}\right)\right]+\frac{\alpha}{2 \pi^{3}} \lambda_{i m n}^{*} \lambda^{j m l} C(R)_{l}{ }^{n}\left[b_{2}-g_{11}\right. \\
& \left.+\frac{1}{2}\left(1+\frac{1}{n}\right)\right]-\frac{1}{8 \pi^{4}} \lambda_{i a c}^{*} \lambda^{j a b} \lambda_{b d e}^{*} \lambda^{c d e}\left[b_{2}-g_{12}+\frac{1}{2}\right]+\frac{1}{16 \pi^{4}} \lambda_{i a b}^{*} \lambda^{k a b} \lambda_{k c d}^{*} \lambda^{j c d} \\
& \left.\times\left[g_{12}-b_{2}\right]\right)+O\left(\alpha^{2} \lambda^{2}, \alpha \lambda^{4}, \lambda^{6}\right)+\text { terms without the Yukawa couplings; }
\end{aligned}
$$




$$
\begin{aligned}
& \left(\widetilde{\gamma}_{\phi}\right)_{i}{ }^{j}(\alpha, \lambda)=-\frac{\alpha}{\pi} C(R)_{i}{ }^{j}+\frac{1}{4 \pi^{2}} \lambda_{i m n}^{*} \lambda^{j m n}+\frac{\alpha}{4 \pi^{3}} \lambda_{l m n}^{*} \lambda^{j m n} C(R)_{i}{ }^{l}\left[g_{12}-g_{11}\right. \\
& \left.-\frac{1}{2}\left(1-\frac{1}{n}\right)\right]+\frac{\alpha}{2 \pi^{3}} \lambda_{i m n}^{*} \lambda^{j m l} C(R)_{l}{ }^{n}\left[g_{12}-g_{11}+\frac{1}{2}\left(1+\frac{1}{n}\right)\right]-\frac{1}{16 \pi^{4}} \lambda_{i a c}^{*} \lambda^{j a b} \\
& \lambda_{b d e}^{*} \lambda^{c d e}+O\left(\alpha^{2}, \alpha \lambda^{4}, \lambda^{6}\right) ; \\
& \widetilde{\gamma}_{V}(\alpha, \lambda)=-\frac{\alpha}{4 \pi}\left(3 C_{2}-T(R)\right)-\frac{\alpha}{16 \pi^{3} r} \lambda_{j m n}^{*} \lambda^{i m n} C(R)_{i}{ }^{j}+O\left(\alpha^{2}, \alpha \lambda^{4}\right),
\end{aligned}
$$

where $b_{2}, g_{11}$, and $g_{12}$ are finite constants which define the subtraction scheme in the considered approximation. We see that for general values of these parameters the NSVZ relation

$$
\left.\frac{\widetilde{\beta}(\alpha, \lambda)}{\alpha^{2}}=-\frac{1}{2 \pi}\left(3 C_{2}-T(R)-2 C_{2} \widetilde{\gamma}_{c}(\alpha, \lambda)-2 C_{2} \widetilde{\gamma}_{V}(\alpha, \lambda)+C(R)_{i}{ }^{j} \widetilde{\gamma}_{\phi}\right)_{j}{ }^{i}(\alpha, \lambda) / r\right)
$$

is not satisfied. However, in the HD+MSL scheme obtained by imposing the conditions (14) with $x_{0}=0$ all these constants vanish, $g_{11}=0 ; g_{12}=0 ; b_{2}=0$ and Eq. (18) is valid. Thus, in the considered approximation for terms containing the Yukawa couplings HD + MSL $=$ NSVZ.

\section{Two-loop anomalous dimension of the Faddeev-Popov ghosts}

The calculations made in [43, 44] allow to verify the terms in Eqs. (12) and (18) which contain anomalous dimensions of the quantum gauge superfield and of the matter superfield. At present, the term containing the ghost anomalous dimension $\widetilde{\gamma}_{c}$ in Eq. (18) has been verified only for the two-loop $\beta$-function and the one-loop anomalous dimension [45]. However, a nontrivial check can be obtain only by comparing the two-loop ghost anomalous dimension and the three-loop $\beta$-function, because only stating from this approximation the scheme dependence becomes essential. The two-loop anomalous dimension of the Faddeev-Popov ghosts has been calculated in [46]. Making this calculation it is very important to take into account that the quantum gauge superfield is renormalized in a non-linear way $[32,33]$. Explicit calculations (for gauge superfield four-point Green function) have been made in $[47,48]$, where it has been demonstrated that the nonlinear terms really appear. In the lowest order it is necessary to take into account nonlinear terms inside the function $\mathcal{F}^{A}(V)=V^{A}+e_{0}^{2} y_{0} G^{A B C D} V^{B} V^{C} V^{D}+\ldots$, where $G^{A B C D}=$ $\left(f^{A K L} f^{B L M} f^{C M N} f^{D N K}+\right.$ permutations of $B, C$, and $\left.D\right) / 6$. According to Refs. $[47,48]$

$$
y_{0}=y+\frac{\alpha}{90 \pi}\left((2+3 \xi) \ln \frac{\Lambda}{\mu}+k_{1}\right)+\ldots
$$

This equation should be taken into account together with the one-loop renormalization of the coupling constant and of the gauge parameter. Then the Faddeev-Popov ghost anomalous dimension defined in terms of the bare couplings for the higher derivative regulators $R(x)=$ $K(x)=1+x^{m} ; F(x)=1+x^{n}$ is given by the scheme-independent expression [46]

$$
\begin{aligned}
& \gamma_{c}\left(\alpha_{0}, \lambda_{0}, \xi_{0}, y_{0}\right)=\frac{\alpha_{0} C_{2}\left(\xi_{0}-1\right)}{6 \pi}-\frac{5 \alpha_{0} y_{0} C_{2}^{2}\left(\xi_{0}-1\right)}{4 \pi}-\frac{\alpha_{0}^{2} C_{2}^{2}}{24 \pi^{2}}\left(\xi_{0}^{2}-1\right) \\
& -\frac{\alpha_{0}^{2} C_{2}^{2}}{4 \pi^{2}}\left(\ln a_{\varphi}+1\right)+\frac{\alpha_{0}^{2} C_{2} T(R)}{12 \pi^{2}}(\ln a+1)+\ldots,
\end{aligned}
$$


where $a \equiv M / \Lambda ; a_{\varphi} \equiv M_{\varphi} / \Lambda$ are ratios of the Pauli-Villars masses to the parameter $\Lambda$. Note that writing Eq. (20) we keep the one-loop $y$-dependence, but omit the dependence on other nonlinearity parameters in the two-loop terms.

The result for the anomalous dimension defined in terms of the renormalized couplings,

$$
\begin{aligned}
& \widetilde{\gamma}_{c}(\alpha, \lambda, \xi, y)=\frac{\alpha C_{2}(\xi-1)}{6 \pi}-\frac{5 \alpha y C_{2}^{2}(\xi-1)}{4 \pi}-\frac{\alpha^{2} C_{2}^{2}}{4 \pi^{2}}\left(\ln a_{\varphi}+1+6 h_{1}-b_{11}\right) \\
& +\frac{\alpha^{2} C_{2} T(R)}{12 \pi^{2}}\left(\ln a+1+6 h_{1}-b_{12}\right)-\frac{\alpha^{2} C_{2}^{2}}{24 \pi^{2}}\left(\xi^{2}-1\right)+\frac{\alpha^{2} C_{2}^{2}}{72 \pi^{2}}\left(4 x_{1}-(\xi-1) k_{1}\right)+\ldots
\end{aligned}
$$

includes some finite constants and is, therefore, scheme-dependent. However, it can be easily verified that in the HD+MSL scheme (for which $b_{11}=b_{12}=0, h_{1}=0, k_{1}=0, x_{1}=0$ ) it coincides with $\gamma_{c}$ after a formal substitution $\alpha \rightarrow \alpha_{0} ; \xi \rightarrow \xi_{0} ; y \rightarrow y_{0}$.

\section{Conclusion}

In the non-Abelian case the perturbative calculations in the lowest orders produce the new form of the NSVZ equation which relates the $\beta$-function to the anomalous dimensions of the quantum gauge superfield, of the Faddeev-Popov ghosts, and of the chiral matter superfields. This new form of the NSVZ relation can be obtained from the usual one with the help of the non-renormalization theorem for the triple ghost-gauge vertices and has the same graphical interpretation as in the Abelian case. In the case of using the higher covariant derivative regularization the new form of the NSVZ relation seems to be valid for RGFs defined in terms of the bare couplings independently of a renormalization prescription. Consequently, the NSVZ scheme for RGFs defined in terms of the renormalized couplings is produced by the HD+MSL prescription. This statement has been verified in the three-loop approximation for terms containing the Yukawa couplings. It is important that in this approximation the scheme dependence of RGFs becomes essential. However, to make a similar check for the other terms, it is necessary to take into account that for supersymmetric theories in higher orders the quantum gauge superfield is renormalized nonlinearly.

\section{References}

[1] M. T. Grisaru, W. Siegel and M. Rocek, Nucl. Phys. B 159, 429 (1979).

[2] V. A. Novikov, M. A. Shifman, A. I. Vainshtein and V. I. Zakharov, Nucl. Phys. B 229, 381 (1983).

[3] D. R. T. Jones, Phys. Lett. B 123, 45 (1983).

[4] V. A. Novikov, M. A. Shifman, A. I. Vainshtein and V. I. Zakharov, Phys. Lett. B 166, 329 (1986); Sov. J. Nucl. Phys. 43, 294 (1986); [Yad. Fiz. 43, 459 (1986)].

[5] M. A. Shifman and A. I. Vainshtein, Nucl. Phys. B 277, 456 (1986); Sov. Phys. JETP 64, 428 (1986); [Zh. Eksp. Teor. Fiz. 91, 723 (1986)].

[6] M. Shifman, Int. J. Mod. Phys. A 33, no.12, 1830009 (2018).

[7] K. V. Stepanyantz, Nucl. Phys. B 909, 316 (2016).

[8] W. Siegel, Phys. Lett. B 84, 193 (1979).

[9] L. V. Avdeev and O. V. Tarasov, Phys. Lett. 112B, 356 (1982).

[10] I. Jack, D. R. T. Jones and C. G. North, Phys. Lett. B 386, 138 (1996).

[11] I. Jack, D. R. T. Jones and C. G. North, Nucl. Phys. B 486, 479 (1997). 
[12] R. V. Harlander, D. R. T. Jones, P. Kant, L. Mihaila and M. Steinhauser, JHEP 0612, 024 (2006).

[13] I. Jack, D. R. T. Jones and A. Pickering, Phys. Lett. B 435, 61 (1998).

[14] D. Kutasov and A. Schwimmer, Nucl. Phys. B 702, 369 (2004).

[15] A. L. Kataev and K. V. Stepanyantz, Theor. Math. Phys. 181, 1531 (2014).

[16] A. A. Slavnov, Nucl. Phys. B 31, 301 (1971).

[17] A. A. Slavnov, Theor.Math.Phys. 13, 1064 (1972); [Teor. Mat. Fiz. 13, 174 (1972)].

[18] A. A. Slavnov, Theor. Math. Phys. 33, 977 (1977); [Teor. Mat. Fiz. 33, 210 (1977)].

[19] V. K. Krivoshchekov, Theor. Math. Phys. 36, 745 (1978); [Teor. Mat. Fiz. 36, 291 (1978)].

[20] P. C. West, Nucl. Phys. B 268, 113 (1986).

[21] K. V. Stepanyantz, Nucl. Phys. B 852, 71 (2011).

[22] K. V. Stepanyantz, JHEP 1408, 096 (2014).

[23] A. L. Kataev and K. V. Stepanyantz, Nucl. Phys. B 875, 459 (2013).

[24] A. L. Kataev and K. V. Stepanyantz, Phys. Lett. B 730, 184 (2014).

[25] A. E. Kazantsev, M. B. Skoptsov and K. V. Stepanyantz, Mod. Phys. Lett. A 32, no.36, 1750194 (2017).

[26] A. L. Kataev, A. E. Kazantsev and K. V. Stepanyantz, Nucl. Phys. B 926, 295 (2018).

[27] K. V. Stepanyantz, "Structure of quantum corrections in $\mathcal{N}=1$ supersymmetric gauge theories," arXiv:1711.09194 [hep-th].

[28] M. Shifman and K. Stepanyantz, Phys. Rev. Lett. 114, 051601 (2015).

[29] M. Shifman and K. V. Stepanyantz, Phys. Rev. D 91, 105008 (2015).

[30] I. V. Nartsev and K. V. Stepanyantz, JHEP 1704, 047 (2017).

[31] I. V. Nartsev and K. V. Stepanyantz, JETP Lett. 105, no.2, 69 (2017).

[32] O. Piguet and K. Sibold, Nucl. Phys. B 197, 257 (1982).

[33] O. Piguet and K. Sibold, Nucl. Phys. B 197, 272 (1982).

[34] I. V. Tyutin, Yad. Fiz. 37, 761 (1983).

[35] S. S. Aleshin, A. E. Kazantsev, M. B. Skoptsov and K. V. Stepanyantz, JHEP 1605, 014 (2016).

[36] D. Dudal, H. Verschelde and S. P. Sorella, Phys. Lett. B 555, 126 (2003).

[37] M. A. L. Capri, D. R. Granado, M. S. Guimaraes, I. F. Justo, L. Mihaila, S. P. Sorella and D. Vercauteren, Eur. Phys. J. C 74, no.4, 2844 (2014).

[38] A. V. Smilga and A. Vainshtein, Nucl. Phys. B 704, 445 (2005).

[39] A. E. Kazantsev and K. V. Stepanyantz, J. Exp. Theor. Phys. 120 no.4, 618 (2015).

[40] A. A. Soloshenko and K. V. Stepanyantz, Theor. Math. Phys. 140, 1264 (2004) [Teor. Mat. Fiz. 140, 437 (2004)]

[41] A. B. Pimenov, E. S. Shevtsova and K. V. Stepanyantz, Phys. Lett. B 686, 293 (2010).

[42] K. V. Stepanyantz, "Factorization of integrals defining the two-loop $\beta$-function for the general renormalizable $\mathrm{N}=1 \mathrm{SYM}$ theory, regularized by the higher covariant derivatives, into integrals of double total derivatives," arXiv:1108.1491 [hep-th].

[43] V. Y. Shakhmanov and K. V. Stepanyantz, Nucl. Phys. B 920, 345 (2017).

[44] A. E. Kazantsev, V. Y. Shakhmanov and K. V. Stepanyantz, JHEP 1804, 130 (2018).

[45] V. Y. Shakhmanov and K. V. Stepanyantz, Phys. Lett. B 776, 417 (2018).

[46] A. E. Kazantsev, M. D. Kuzmichev, N. P. Meshcheriakov, S. V. Novgorodtsev, I. E. Shirokov, M. B. Skoptsov and K. V. Stepanyantz, JHEP 1806, 020 (2018).

[47] J. W. Juer and D. Storey, Phys. Lett. 119B, 125 (1982).

[48] J. W. Juer and D. Storey, Nucl. Phys. B 216, 185 (1983). 